\title{
LOCAL MOMENT MATCHING AND $S$-CONVEX EXTREMA
}

\author{
BY
}

\author{
Cindy Courtois And Michel Denuit
}

\begin{abstract}
The paper is devoted to the local moment matching method and its links with the discrete version of the $s$-convex extremal distributions. It is well-known that the local moment matching method can produce some negative masses. Connecting the local moment matching method to the discrete $s$-convex extrema gives an explicit criterion that explains why (and says when) the local moment matching method gives some negative mass.
\end{abstract}

\section{KEYWORDS}

Discretisation, local moment matching, s-convex order.

\section{INTRODUCTION}

Given any risk $X$ with support included in $[0,+\infty)$, a relevant problem in actuarial applications is to arithmetize it, i.e. to choose a span $\Delta>0$ and try to determine a risk $X_{\Delta}$ with support points $i \Delta, i=0,1,2, \ldots$ that shares some common characteristics with $X$ and that will serve for the numerical computations. This operation is required by the majority of iterative algorithms (like Panjer's algorithm for instance).

In the actuarial literature several methods have been proposed to discretize claim amount distributions, e.g., by GERBER (1982), PANJER \& LUTEK (1983), WALHIN \& PARIS (1998) and VILAR (2000). The local moment matching method (LMM in short) proposed by GERBER (1982) constructs discrete equispaced distributions that matches some moments locally. The idea is to replace the probability mass of $F_{X}$ over intervals $\left(a_{k}, b_{k}\right]$ by pointmasses located at $x_{i}^{(k)}=a_{k}+$ $i \Delta(i=0,1, \ldots, n)$, where $\Delta=\left(b_{k}-a_{k}\right) / n$ and the integer $n$ depends on the number of moments to be matched. The method tries thus to calculate the $n+1$ masses $p_{0}^{(k)}, p_{1}^{(k)}, \ldots p_{n}^{(k)}$ located at the points $x_{0}^{(k)}, x_{1}^{(k)}, \ldots, x_{n}^{(k)}$ which are solutions of the linear system

$$
\sum_{i=0}^{n}\left(a_{k}+i \Delta\right)^{r} p_{i}^{(k)}=\int_{a_{k}}^{b_{k}} x^{r} d F_{X}(x), \quad r=0,1, \ldots, n ;
$$


the unique solution of this system being

$$
p_{i}^{(k)}=\int_{a_{k}}^{b_{k}}\left(\prod_{j \neq i} \frac{x-a_{k}-j \Delta}{(i-j) \Delta}\right) d F_{X}(x), i=0,1, \ldots, n .
$$

In practice, this system has to be solved in each of the intervals $(0, n \Delta],(n \Delta, 2 n \Delta]$, $(2 n \Delta, 3 n \Delta], \ldots$ corresponding to $a_{0}=0, a_{1}=n \Delta, a_{2}=2 n \Delta, \ldots$ The final probabilities of the discretized distribution are the point masses obtained as solutions of the previous system in each interval, summed for the end points of each interval.

In this paper, moment-based discretisation methods are studied and linked to discrete stochastic extrema. This permits to give simple conditions to verify whether the local moment matching method gives negative masses for a given span $\Delta>0$. The paper is organized as follows. First, we recall some notions about $s$-convex extrema and moment spaces. Then these extrema are used in connection with the local moment matching method. In particular, we derive explicit expressions for the admissibility of the local moment matching method. Finally, numerical examples are given.

\section{DiSCRETE $S$-CONVEX ORDERINGS AND MOMENT SPACES}

DENUIT \& LEFÈVRE (1997) introduced the discrete s-convex orderings among arithmetic random variables valued in some set $\mathcal{D}_{n}=\left\{e_{0}+i h: i=0, \ldots, n\right\}$ of $(n+1)$ evenly-spaced points, with minimum $e_{0}$ and separation parameter $h>0$ say. Here $s$ is any non-negative integer smaller or equal to $n$. These orderings are defined as follows. Let $\Delta_{h}$ be the forward difference operator with increment $h$ defined for each function $\phi: \mathcal{D}_{n} \rightarrow \mathbb{R}$ by $\Delta_{h} \phi\left(e_{0}+i h\right)=\phi\left(e_{0}+(i+1) h\right)-\phi\left(e_{0}+i h\right)$ for all $i=0, \ldots, n-1$. Let $\Delta_{h}^{k}, k \geq 1$, be the $k$-th forward difference operator defined recursively by $\Delta_{h}^{k} \phi\left(e_{0}+i h\right)=\Delta_{h}^{k-1} \phi\left(e_{0}+(i+1) h\right)-\Delta_{h}^{k-1} \phi\left(e_{0}+i h\right)$ for all $i=0, \ldots, n-k$ (by convention, $\Delta_{h}^{1} \phi \equiv \Delta \phi$ and $\left.\Delta_{h}^{0} \phi \equiv \phi\right)$. If $X$ and $Y$ are two random variables valued in $\mathcal{D}_{n}, X$ is said to be smaller than $Y$ with respect to the discrete $s$-convex order if $\mathbb{E}[\phi(X)] \leq \mathbb{E}[\phi(Y)]$ for all the functions $\phi: \mathcal{D}_{n} \rightarrow \mathbb{R}$ such that $\Delta_{h}^{s} \phi\left(e_{0}+i h\right) \geq 0$ for all $i=0, \ldots, n-s$. In such a case, we write $X \preceq_{s-c x}^{\mathcal{D}_{n}} Y$. Clearly,

$$
X \preceq_{s-c x}^{\mathcal{D}_{n}} Y \Rightarrow \mathbb{E}\left[X^{k}\right]=\mathbb{E}\left[Y^{k}\right] \text { for } k=1,2, \ldots, s-1
$$

Thus, if $X \preceq_{s-c x}^{\mathcal{D}_{n}} Y$ then the $s-1$ first moments of $X$ and $Y$ necessarily match. Consequently, the ordering relation $\preceq_{s-c x}^{\mathcal{D}_{n}}$ can only be used to compare the random variables with the same first $s-1$ moments. This motivates to introduce the moment space $\mathcal{M}_{s}\left(\mathcal{D}_{n} ; \mu_{1}, \mu_{2}, \ldots, \mu_{s-1}\right)$ which contains all random variables valued on $\mathcal{D}_{n}$ such that the first $s-1$ moments are fixed to $\mu_{k}(k=1, \ldots, s-1 ; s \geq 1)$.

Now, suppose that the moment sequence $\left(\mu_{1}, \mu_{2}, \ldots, \mu_{s-1}\right)$ is such that $\mathcal{M}_{s}\left(\mathcal{D}_{n}\right.$; $\left.\mu_{1}, \mu_{2}, \ldots, \mu_{s-1}\right)$ is not void. Conditions for such a space to be non void can be 
found in KARLIN \& STUDDEN (1966): $\mathcal{M}_{s}\left(\mathcal{D}_{n} ; \mu_{1}, \mu_{2}, \ldots, \mu_{s-1}\right)$ is non void if, and only if, the point $\left(\mu_{1}, \ldots, \mu_{s-1}\right)$ is an element of the convex hull of $\left\{\left(\theta, \theta^{2}, \ldots\right.\right.$, $\left.\left.\theta^{s-1}\right) \mid \theta \in \mathcal{D}_{n}\right\}$. As in DenUit, De Vylder \& LefÈVRe (1999) and HürLimanN (2005), let us put $\mu_{0} \equiv 1$ and for $j=1,2, \ldots$, and $x, y, z \in \mathbb{R}$, let us define

$$
\mu_{j, x}=x \mu_{j-1}-\mu_{j}, \quad \mu_{j, x, y}=y \mu_{j-1, x}-\mu_{j, x} \quad \text { and } \quad \mu_{j, x, y, z}=z \mu_{j-1, x, y}-\mu_{j, x, y} .
$$

With these notations, if $e_{i}=e_{0}+i h(i=0,1, \ldots, n)$, the conditions for $\mathcal{M}_{s}\left(\mathcal{D}_{n}\right.$; $\left.\mu_{1}, \mu_{2}, \ldots, \mu_{s-1}\right) \neq \emptyset, s=2,3,4$, are

- $s=2: \mu_{1, e_{0}} \leq 0$ and $\mu_{1, e_{n}} \geq 0$;

- $s=3: \mu_{2, e_{0}, e_{n}} \leq 0$ and $\mu_{2, e_{i}, e_{i+1}} \geq 0$ for $i=0, \ldots, n-1$;

- $s=4: \mu_{3, e_{0}, e_{i}, e_{i+1}} \leq 0$ for $i=1, \ldots, n-1$, and $\mu_{3, e_{i}, e_{i+1}, e_{n}} \geq 0$ for $i=0, \ldots, n-2$.

Under such conditions, the moment space contains a minimum random variable $X_{\min }^{(s)}$ and a maximum random variable $X_{\max }^{(s)}$ with respect to $\preceq_{s-c x}^{\mathcal{D}_{n}}$, i.e. such that

$$
X_{\min }^{(s)} \preceq_{s-c x}^{\mathcal{D}_{n}} X \preceq_{s-c x}^{\mathcal{D}_{n}} X_{\max }^{(s)} \text { for all } X \in \mathcal{M}_{s}\left(\mathcal{D}_{n} ; \mu_{1}, \mu_{2}, \ldots, \mu_{s-1}\right) .
$$

Extrema with respect to the discrete version of the $s$-convex orders have been derived by Denuit \& LefÈvre (1997), Denuit, LefĖvre \& Mesfioui (1999) and Courtois, Denuit \& Van Bellegem (2006) for $s \geq 1$.

\section{ARIthmetiZATION AND DISCRETE S-CONVEX EXTREMA}

\subsection{One moment known}

On each interval $I_{k}=(k \Delta,(k+1) \Delta], k=0,1,2, \ldots$, the first local moment of $X$ is

$$
\mu_{1}^{(k)}=\int_{k \Delta}^{(k+1) \Delta} x d F_{X}(x) .
$$

We obviously have that

$$
k \Delta \mu_{0}^{(k)}<\mu_{1}^{(k)}<(k+1) \Delta \mu_{0}^{(k)}, \quad k=0,1,2, \ldots,
$$

where $\mu_{0}^{(k)}=\int_{k \Delta}^{(k+1) \Delta} d F_{X}(x)$ is the probability mass over the interval $I_{k}$. Then, for $k=1,2, \ldots$, the only random variable with support $\{k \Delta,(k+1) \Delta\}$ and mean $\mu_{1}^{(k)} / \mu_{0}^{(k)}$ is the 2-convex maximum

$$
X_{k, \max }^{(2)}= \begin{cases}k \Delta & \text { with probability } \frac{(k+1) \Delta \mu_{0}^{(k)}-\mu_{1}^{(k)}}{\Delta \mu_{0}^{(k)}}, \\ (k+1) \Delta & \text { with probability } \frac{\mu_{1}^{(k)}-k \Delta \mu_{0}^{(k)}}{\Delta \mu_{0}^{(k)}} .\end{cases}
$$


The weights associated to $k \Delta$ and $(k+1) \Delta$ are thus respectively

$$
\begin{aligned}
& w_{k}^{+}=\frac{(k+1) \Delta \mu_{0}^{(k)}-\mu_{1}^{(k)}}{\Delta} \\
& w_{k}^{-}=\frac{\mu_{1}^{(k)}-k \mu_{0}^{(k)}}{\Delta} .
\end{aligned}
$$

Consequently, if we denote by $X_{\Delta}^{(2)}$ the arithmetized risk based on the knowledge of one moment of $X$, we have $\mathbb{P}\left[X_{\Delta}^{(2)}=0\right]=F_{X}(0)+w_{0}^{+}$and, for $k=1,2$, $\ldots, \mathbb{P}\left[X_{\Delta}^{(2)}=k \Delta\right]=w_{k}^{-}+w_{k}^{+}$.

\subsection{Two moments known}

On each interval $I_{k}=(k \Delta,(k+2) \Delta], k=0,2,4, \ldots$, the first two local moments of $X$ over $I_{k}$ are

$$
\mu_{1}^{(k)}=\int_{k \Delta}^{(k+2) \Delta} x d F_{X}(x) \text { and } \mu_{2}^{(k)}=\int_{k \Delta}^{(k+2) \Delta} x^{2} d F_{X}(x) .
$$

Let us now consider the set of all the probability distributions with support $\{k \Delta,(k+1) \Delta,(k+2) \Delta\}$ and first two moments $\mu_{1}^{(k)} / \mu_{0}^{(k)}$ and $\mu_{2}^{(k)} / \mu_{0}^{(k)}$. The conditions for that set to be not void are

$$
\left\{\begin{array}{l}
k \Delta \mu_{0}^{(k)}<\mu_{1}^{(k)}<(k+2) \Delta \mu_{0}^{(k)}, \\
\mu_{2}^{(k)}<2 \Delta(k+1) \mu_{1}^{(k)}-k(k+2) \Delta^{2} \mu_{0}^{(k)}, \\
\mu_{2}^{(k)}>\Delta(2 k+1) \mu_{1}^{(k)}-k(k+1) \Delta^{2} \mu_{0}^{(k)}, \\
\mu_{2}^{(k)}>\Delta(2 k+3) \mu_{1}^{(k)}-(k+1)(k+2) \Delta^{2} \mu_{0}^{(k)} .
\end{array}\right.
$$

As $\int_{k \Delta}^{(k+2) \Delta}(x-k \Delta) d F(x) \geq 0, \quad \int_{k \Delta}^{(k+2) \Delta}((k+2) \Delta-x) d F(x) \geq 0$ and $\int_{k \Delta}^{(k+2) \Delta}(x-k \Delta)((k+2) \Delta-x) d F(x) \geq 0$, system (2) reduces to its last two equations, that is

$$
\left\{\begin{array}{l}
\int_{k \Delta}^{(k+2) \Delta}(x-k \Delta)(x-(k+1) \Delta) d F(x)>0 \\
\int_{k \Delta}^{(k+2) \Delta}(x-(k+1) \Delta)(x-(k+2) \Delta) d F(x)>0 .
\end{array}\right.
$$

The only random variable with support $\{k \Delta,(k+1) \Delta,(k+2) \Delta\}$ and first moments $\mu_{1}^{(k)} / \mu_{0}^{(k)}$ and $\mu_{2}^{(k)} / \mu_{0}^{(k)}$ is the 3 -convex maximum 


$$
X_{k, \max }^{(3)}= \begin{cases}k \Delta \quad \text { with probability } \frac{\mu_{2}^{(k)}-\mu_{1}^{(k)}(2 k+3) \Delta+\mu_{0}^{(k)}(k+1)(k+2) \Delta^{2}}{\mu_{0}^{(k)} 2 \Delta^{2}}, \\ (k+1) \Delta \text { with probability } \frac{-\mu_{2}^{(k)}+\mu_{1}^{(k)}(2 k+2) \Delta-\mu_{0}^{(k)} k(k+2) \Delta^{2}}{\mu_{0}^{(k)} \Delta^{2}}, \\ (k+2) \Delta \text { with probability } \frac{\mu_{2}^{(k)}-\mu_{1}^{(k)}(2 k+1) \Delta+\mu_{0}^{(k)} k(k+1) \Delta^{2}}{\mu_{0}^{(k)} 2 \Delta^{2}} .\end{cases}
$$

The weights associated to $k \Delta,(k+1) \Delta$ and $(k+2) \Delta$ are thus respectively

$$
\begin{aligned}
& w_{k}^{+}=\frac{\mu_{2}^{(k)}-\mu_{1}^{(k)}(2 k+3) \Delta+\mu_{0}^{(k)}(k+1)(k+2) \Delta^{2}}{2 \Delta^{2}} \\
& w_{k+1}=\frac{-\mu_{2}^{(k)}+\mu_{1}^{(k)}(2 k+2) \Delta-\mu_{0}^{(k)} k(k+2) \Delta^{2}}{\Delta^{2}} \\
& w_{k+2}^{-}=\frac{\mu_{2}^{(k)}-\mu_{1}^{(k)}(2 k+1) \Delta+\mu_{0}^{(k)} k(k+1) \Delta^{2}}{2 \Delta^{2}} .
\end{aligned}
$$

Consequently, if $X_{\Delta}^{(3)}$ is the arithmetized risk based on the knowledge of the first two moments of $X$, we have $\mathbb{P}\left[X_{\Delta}^{(3)}=0\right]=F_{X}(0)+w_{0}^{+}$and, for $k=0,2,4, \ldots$, $\mathbb{P}\left[X_{\Delta}^{(3)}=(k+1) \Delta\right]=w_{k+1}$ and $\mathbb{P}\left[X_{\Delta}^{(3)}=(k+2) \Delta\right]=w_{k+2}^{-}+w_{k+2}^{+}$.

\subsection{Three moments known}

On each interval $I_{k}=(k \Delta,(k+3) \Delta], k=0,3,6, \ldots$, the first local moments of $X$ over $I_{k}$ are

$\mu_{1}^{(k)}=\int_{k \Delta}^{(k+3) \Delta} x d F_{X}(x), \mu_{2}^{(k)}=\int_{k \Delta}^{(k+3) \Delta} x^{2} d F_{X}(x)$, and $\mu_{3}^{(k)}=\int_{k \Delta}^{(k+3) \Delta} x^{3} d F_{X}(x)$.

Let us now consider the set of all the probability distributions with support $\{k \Delta,(k+1) \Delta,(k+2) \Delta,(k+3) \Delta\}$ and first three moments $\mu_{1}^{(k)} / \mu_{0}^{(k)}, \mu_{2}^{(k)} / \mu_{0}^{(k)}$ and $\mu_{3}^{(k)} / \mu_{0}^{(k)}$. The conditions for that set to be not void are

$$
\left\{\begin{array}{l}
k \Delta \mu_{0}^{(k)}<\mu_{1}^{(k)}<(k+3) \Delta \mu_{0}^{(k)}, \\
\mu_{2}^{(k)}<\Delta(2 k+3) \mu_{1}^{(k)}-k(k+3) \Delta^{2} \mu_{0}^{(k)}, \\
\mu_{2}^{(k)}>\Delta(2 k+1) \mu_{1}^{(k)}-k(k+1) \Delta^{2} \mu_{0}^{(k)} \\
\mu_{2}^{(k)}>\Delta(2 k+3) \mu_{1}^{(k)}-(k+1)(k+2) \Delta^{2} \mu_{0}^{(k)}, \\
\mu_{2}^{(k)}>\Delta(2 k+5) \mu_{1}^{(k)}-(k+2)(k+3) \Delta^{2} \mu_{0}^{(k)} \\
\mu_{3}^{(k)}<\Delta(3 k+4) \mu_{2}^{(k)}-\Delta^{2}\left(3 k^{2}+8 k+3\right) \mu_{1}^{(k)}+k(k+1)(k+3) \Delta^{3} \mu_{0}^{(k)}, \\
\mu_{3}^{(k)}<\Delta(3 k+6) \mu_{2}^{(k)}-\Delta^{2}\left(3 k^{2}+12 k+11\right) \mu_{1}^{(k)}+(k+1)(k+2)(k+3) \Delta^{3} \mu_{0}^{(k)}, \\
\mu_{3}^{(k)}>3 \Delta(k+1) \mu_{2}^{(k)}-\Delta^{2}\left(3 k^{2}+6 k+2\right) \mu_{1}^{(k)}+k(k+1)(k+2) \Delta^{3} \mu_{0}^{(k)}, \\
\mu_{3}^{(k)}>\Delta(3 k+5) \mu_{2}^{(k)}-\Delta^{2}\left(3 k^{2}+10 k+6\right) \mu_{1}^{(k)}+k(k+2)(k+3) \Delta^{3} \mu_{0}^{(k)}
\end{array}\right.
$$


As $\int_{k \Delta}^{(k+3) \Delta}(x-k \Delta) d F(x) \geq 0, \int_{k \Delta}^{(k+3) \Delta}((k+3) \Delta-x) d F(x) \geq 0$ and $\int_{k \Delta}^{(k+3) \Delta}(x-k \Delta)((k+3) \Delta-x) d F(x) \geq 0$, system (3) reduces to

$$
\left\{\begin{array}{l}
\int_{k \Delta}^{(k+3) \Delta}(x-k \Delta)(x-(k+1) \Delta) d F(x)>0, \\
\int_{k \Delta}^{(k+3) \Delta}(x-(k+1) \Delta)(x-(k+2) \Delta) d F(x)>0, \\
\int_{k \Delta}^{(k+3) \Delta}(x-(k+2) \Delta)(x-(k+3) \Delta) d F(x)>0, \\
\int_{k \Delta}^{(k+3) \Delta}(x-k \Delta)(x-(k+1) \Delta)((k+3) \Delta-x) d F(x)>0, \\
\int_{k \Delta}^{(k+3) \Delta}(x-(k+1) \Delta)(x-(k+2) \Delta)((k+3) \Delta-x) d F(x)>0, \\
\int_{k \Delta}^{(k+3) \Delta}(x-k \Delta)(x-(k+1) \Delta)(x-(k+2) \Delta) d F(x)>0, \\
\int_{k \Delta}^{(k+3) \Delta}(x-k \Delta)(x-(k+2) \Delta)(x-(k+3) \Delta) d F(x)>0 .
\end{array}\right.
$$

The only random variable with support $\{k \Delta,(k+1) \Delta,(k+2) \Delta,(k+3) \Delta\}$ and first moments $\mu_{1}^{(k)} / \mu_{0}^{(k)}, \mu_{2}^{(k)} / \mu_{0}^{(k)}$ and $\mu_{3}^{(k)} / \mu_{0}^{(k)}$ is the 4-convex maximum

$$
X_{k, \max }^{(4)}=\left\{\begin{array}{l}
k \Delta \quad \text { with probability } \frac{-\mu_{3}^{(k)}+\Delta(3 k+6) \mu_{2}^{(k)}-\Delta^{2}\left(3 k^{2}+12 k+11\right) \mu_{1}^{(k)}+(k+1)(k+2)(k+3) \Delta^{3} \mu_{0}^{(k)}}{6 \mu_{0}^{(k)} \Delta^{3}}, \\
(k+1) \Delta \text { with probability } \frac{\mu_{3}^{(k)}-\Delta(3 k+5) \mu_{2}^{(k)}+\Delta^{2}\left(3 k^{2}+10 k+6\right) \mu_{1}^{(k)}-k(k+2)(k+3) \Delta^{3} \mu_{0}^{(k)}}{2 \mu_{0}^{(k)} \Delta^{3}}, \\
(k+2) \Delta \text { with probability } \frac{-\mu_{3}^{(k)}+\Delta(3 k+4) \mu_{2}^{(k)}-\Delta^{2}\left(3 k^{2}+8 k+3\right) \mu_{1}^{(k)}+k(k+1)(k+3) \Delta^{3} \mu_{0}^{(k)}}{2 \mu_{0}^{(k)} \Delta^{3}}, \\
(k+3) \Delta \text { with probability } \frac{\mu_{3}^{(k)}-3 \Delta(k+1) \mu_{2}^{(k)}+\Delta^{2}\left(3 k^{2}+6 k+2\right) \mu_{1}^{(k)}-k(k+1)(k+2) \Delta^{3} \mu_{0}^{(k)}}{6 \mu_{n}^{(k)} \Delta^{3}} .
\end{array}\right.
$$

The weights associated to $k \Delta,(k+1) \Delta,(k+2) \Delta$ and $(k+3) \Delta$ are thus respectively

$$
\begin{aligned}
& w_{k}^{+}=\frac{-\mu_{3}^{(k)}+\Delta(3 k+6) \mu_{2}^{(k)}-\Delta^{2}\left(3 k^{2}+12 k+11\right) \mu_{1}^{(k)}+(k+1)(k+2)(k+3) \Delta^{3} \mu_{0}^{(k)}}{6 \mu_{0}^{(k)} \Delta^{3}} \\
& w_{k+1}=\frac{\mu_{3}^{(k)}-\Delta(3 k+5) \mu_{2}^{(k)}+\Delta^{2}\left(3 k^{2}+10 k+6\right) \mu_{1}^{(k)}-k(k+2)(k+3) \Delta^{3} \mu_{0}^{(k)}}{2 \mu_{0}^{(k)} \Delta^{3}} \\
& w_{k+2}=\frac{-\mu_{3}^{(k)}+\Delta(3 k+4) \mu_{2}^{(k)}-\Delta^{2}\left(3 k^{2}+8 k+3\right) \mu_{1}^{(k)}+k(k+1)(k+3) \Delta^{3} \mu_{0}^{(k)}}{2 \mu_{0}^{(k)} \Delta^{3}} \\
& w_{k+3}^{-}=\frac{\mu_{3}^{(k)}-3 \Delta(k+1) \mu_{2}^{(k)}+\Delta^{2}\left(3 k^{2}+6 k+2\right) \mu_{1}^{(k)}-k(k+1)(k+2) \Delta^{3} \mu_{0}^{(k)}}{6 \mu_{n}^{(k)} \Delta^{3}} .
\end{aligned}
$$


Consequently, if $X_{\Delta}^{(4)}$ is the arithmetized risk based on the knowledge of the first three moments of $X$, we have $\mathbb{P}\left[X_{\Delta}^{(4)}=0\right]=F_{X}(0)+w_{0}^{+}$and, for $k=0,3,6, \ldots$, $\mathbb{P}\left[X_{\Delta}^{(4)}=(k+1) \Delta\right]=w_{k+1}, \mathbb{P}\left[X_{\Delta}^{(4)}=(k+2) \Delta\right]=w_{k+2}$, and $\mathbb{P}\left[X_{\Delta}^{(4)}=(k+3) \Delta\right]=$ $w_{k+3}^{-}+w_{k+3}^{+}$.

\subsection{General case}

Given any risk $X$ with support included in $[0,+\infty)$ and probability distribution function denoted by $F_{X}$, the problem addressed in this section is to choose a span $\Delta>0$ and try to determine the arithmetic distribution with support points $i \Delta, i=0,1,2, \ldots$, that has the property of equating $s-1$ moments with $F_{X}$.

To that end, we will proceed as follows. On each of the intervals $I_{k}=(k(s-1)$ $\Delta,(k+1)(s-1) \Delta](k=0,1,2, \ldots)$, let us denote by $\mu_{j}^{(k)}, j=0,1, \ldots, s-1$, the local moment of $X$ on $I_{k}$, that is

$$
\mu_{j}^{(k)}=\int_{k(s-1) \Delta}^{(k+1)(s-1) \Delta} x^{j} d F_{X}(x) .
$$

For $j=0, \mu_{0}^{(k)}$ is the probability mass for the interval $I_{k}$ so that $\mu_{j}^{(k)} / \mu_{0}^{(k)}$ are the conditional moments. Furthermore, we suppose that the moment space $\mathcal{M}_{s}\left(\mathcal{E}_{s}^{(k)} ; \frac{\mu_{1}^{(k)}}{\mu_{0}^{(k)}}, \frac{\mu_{2}^{(k)}}{\mu_{0}^{(k)}}, \ldots, \frac{\mu_{s-1}^{(k)}}{\mu_{0}^{(k)}}\right)$ of all the random variables valued in $\mathcal{E}_{\mu_{s}^{(k)}}^{(k)}=k(s-1)$ $\Delta+\{0, \Delta, \ldots,(s-1) \Delta\}$ and with prescribed first $s-1$ moments $\frac{\mu_{j}^{(k)}}{\mu_{0}^{(k)}}(j=1,2, \ldots$, $s-1)$ is non void. Such conditions are recalled in Section 2. Then, the unique element of $\mathcal{M}_{s}\left(\mathcal{E}_{s}^{(k)} ; \frac{\mu_{1}^{(k)}}{\mu_{0}^{(k)}}, \frac{\mu_{2}^{(k)}}{\mu_{0}^{(k)}}, \ldots, \frac{\mu_{s-1}^{(k)}}{\mu_{0}^{(k)}}\right)$ is the $s$-convex maximum. The momentbased discretisation method thus amounts to choose, on each interval $I_{k}$, the extremal random variable with support points $k(s-1) \Delta,(k(s-1)+1) \Delta, \ldots$, $(k+1)(s-1) \Delta$.

\section{Numerical APPLiCATIONS}

In this section, we will present numerical applications of the moment-based discretisation method presented in Section 3. Specifically, we study the admissibility of the local moment matching method when discretizing Exponential, Gamma, Pareto and Log-Normal distributions for various values of their parameters. These parametric models have the following moments as functions of their parameters

- $X \sim \operatorname{Exp}(\theta) \Rightarrow \mathbb{E}\left[X^{k}\right]=\frac{k !}{\theta^{k}}, \quad k=1,2, \ldots$;

- $X \sim \operatorname{Gam}(a, \tau) \Rightarrow \mathbb{E}\left[X^{k}\right]=\frac{1}{\tau^{k}} \prod_{i=0}^{k-1}(\alpha+i), \quad k=1,2, \ldots$;

- $X \sim \operatorname{Par}(a, \theta) \Rightarrow \mathbb{E}\left[X^{k}\right]=\frac{k ! \alpha \theta^{k}(\alpha-k-1) !}{\alpha !}$ if $\alpha>k, k=1,2, \ldots$;

- $X \sim \mathcal{L} \mathcal{N} \operatorname{or}(\mu, \sigma) \Rightarrow \mathbb{E}\left[X^{k}\right]=\exp \left(\mu k+\frac{\sigma^{2} k^{2}}{2}\right), \quad k=1,2, \ldots$ 
In Tables 1, 2, 3, 4, 5, 6, 7, 8 and 9, we study the admissibility (i.e. when all the derived masses are positive) of the local moment matching method in term of the number of moments used and the chosen span. Since the local moment matching method is applicable only if the random variable to be discretized has a bounded support, the admissibility of the method is tested with respect to arbitrary fixed upper limits of the supports, denoted by $N$ (see Tables 1, 2, 3, 4). We see from these examples that there is a maximum span $\Delta_{\max }>0$ for which, for all given span $\Delta>0$ such that $(s-1) \Delta<N$ (i.e. there is more than one interval $I_{k}$ in Section 3) and $\Delta \leq \Delta_{\max }$, the local moment matching method leads to a discretisation of the distribution of interest with positive masses. In view of Tables 1, 2, 3 and 4, it appears that the chosen value for $N$ does not seem to have any impact on the admissibility of the span $\Delta$. Therefore, we work with $N=5$ in the subsequent tables. We see from Tables 5-9 that the admissibility pattern is always the same: the discretization becomes possible provided $\Delta$ becomes small enough. Moreover, a close examination of Tables 5-9 reveals that it seems to be easier to discretize these distributions as they become "larger", that is, as their expected value increases. In the Negative Exponential

TABLE 1

ADMISSIBILITY OF THE LOCAL MOMENT MATCHING METHOD WITH TWO OR THREE MOMENTS KNOWN, $\theta_{E X P}=0.6, \alpha_{G A M}=2, \tau_{G A M}=1, \alpha_{P A R}=4, \theta_{P A R}=5, \mu_{L N}=0, \sigma_{L N}=1.5$ and $N=2.5$.

\begin{tabular}{c|c|cccc}
\hline \hline Upper limit & Span & Exponential & Gamma & Pareto & Log-Normal \\
\hline \multirow{2}{*}{$s=3$} & 2 & Yes & Yes & No & No \\
& 1.75 & Yes & Yes & Yes & Yes \\
& 1.5 & Yes & Yes & Yes & Yes \\
& 1.25 & Yes & Yes & Yes & Yes \\
\hline \multirow{5}{*}{$s=4$} & 1 & Yes & Yes & Yes & Yes \\
& 0.75 & Yes & Yes & Yes & Yes \\
& 0.5 & Yes & Yes & Yes & Yes \\
& 0.25 & Yes & Yes & Yes & Yes \\
& 0.1 & Yes & Yes & Yes & Yes \\
& 0.05 & Yes & Yes & Yes & Yes \\
& 1.75 & Yes & Yes & No & No \\
& 1.5 & Yes & Yes & No & No \\
& 1.25 & Yes & Yes & Yes & Yes \\
& 1 & Yes & Yes & Yes & Yes \\
& 0.75 & Yes & Yes & Yes & Yes \\
\hline \hline & 0.5 & Yes & Yes & Yes & Yes \\
& 0.25 & Yes & Yes & Yes & Yes \\
& 0.1 & Yes & Yes & Yes & Yes \\
& 0.05 & Yes & Yes & Yes & Yes \\
& & Yes & Yes & Yes & Yes \\
\hline \hline
\end{tabular}


case, for instance, we see from Table 5 that smaller spans are admissible as $\theta$ decreases.

All the examples discussed so far consist in discretizing a continuous distribution. Let us now apply this approach to arithmetize a discrete distribution with an irregularly spaced support. To this end, we consider the hypothetical claim severity distribution given in WALHIN \& PARIS (1998):

\begin{tabular}{l|ccccccccccc} 
Support points & 0 & 7 & 12 & 17 & 21 & 23 & 28 & 39 & 46 & 53 & 67 \\
\hline Probabilities & 0.05 & 0.1 & 0.15 & 0.05 & 0.05 & 0.05 & 0.1 & 0.1 & 0.1 & 0.15 & 0.1
\end{tabular}

TABLE 2

ADMISSIBILITY OF THE LOCAL MOMENT MATCHING METHOD WITH TWO OR THREE MOMENTS KNOWN, $\theta_{E X P}=0.6, \alpha_{G A M}=2, \tau_{G A M}=1, \alpha_{P A R}=4, \theta_{P A R}=5, \mu_{L N}=0, \sigma_{L N}=1.5$ and $N=5$.

\begin{tabular}{|c|c|c|c|c|c|}
\hline Upper limit & Span & Exponential & Gamma & Pareto & Log-Normal \\
\hline \multirow[t]{14}{*}{$s=3$} & 3 & No & No & No & No \\
\hline & 2.75 & No & No & No & No \\
\hline & 2.5 & No & Yes & No & No \\
\hline & 2.25 & No & No & Yes & Yes \\
\hline & 2 & Yes & No & Yes & Yes \\
\hline & 1.75 & Yes & No & Yes & Yes \\
\hline & 1.5 & Yes & Yes & Yes & Yes \\
\hline & 1.25 & Yes & Yes & Yes & Yes \\
\hline & 1 & Yes & Yes & Yes & Yes \\
\hline & 0.75 & Yes & Yes & Yes & Yes \\
\hline & 0.5 & Yes & Yes & Yes & Yes \\
\hline & 0.25 & Yes & Yes & Yes & Yes \\
\hline & 0.1 & Yes & Yes & Yes & Yes \\
\hline & 0.05 & Yes & Yes & Yes & Yes \\
\hline \multirow[t]{14}{*}{$s=4$} & 3 & No & No & No & No \\
\hline & 2.75 & No & No & No & No \\
\hline & 2.5 & No & No & No & No \\
\hline & 2.25 & No & Yes & No & No \\
\hline & 2 & Yes & Yes & No & No \\
\hline & 1.75 & Yes & Yes & No & No \\
\hline & 1.5 & Yes & No & Yes & Yes \\
\hline & 1.25 & Yes & Yes & Yes & Yes \\
\hline & 1 & Yes & Yes & Yes & Yes \\
\hline & 0.75 & Yes & Yes & Yes & Yes \\
\hline & 0.5 & Yes & Yes & Yes & Yes \\
\hline & 0.25 & Yes & Yes & Yes & Yes \\
\hline & 0.1 & Yes & Yes & Yes & Yes \\
\hline & 0.05 & Yes & Yes & Yes & Yes \\
\hline
\end{tabular}


TABLE 3

ADMISSIBILITY OF THE LOCAL MOMENT MATCHING METHOD WITH TWO OR THREE MOMENTS KNOWN, $\theta_{E X P}=0.6, \alpha_{G A M}=2, \tau_{G A M}=1, \alpha_{P A R}=4, \theta_{P A R}=5, \mu_{L N}=0, \sigma_{L N}=1.5$ and $N=7.5$.

\begin{tabular}{|c|c|c|c|c|c|}
\hline Upper limit & Span & Exponential & Gamma & Pareto & Log-Normal \\
\hline \multirow{18}{*}{$s=3$} & 4 & No & No & No & No \\
\hline & 3.75 & No & No & No & No \\
\hline & 3.5 & No & No & No & No \\
\hline & 3.25 & No & No & No & No \\
\hline & 3 & No & No & No & Yes \\
\hline & 2.75 & No & No & No & Yes \\
\hline & 2.5 & No & No & No & Yes \\
\hline & 2.25 & No & No & Yes & Yes \\
\hline & 2 & Yes & No & Yes & Yes \\
\hline & 1.75 & Yes & No & Yes & Yes \\
\hline & 1.5 & Yes & Yes & Yes & Yes \\
\hline & 1.25 & Yes & Yes & Yes & Yes \\
\hline & 1 & Yes & Yes & Yes & Yes \\
\hline & 0.75 & Yes & Yes & Yes & Yes \\
\hline & 0.5 & Yes & Yes & Yes & Yes \\
\hline & 0.25 & Yes & Yes & Yes & Yes \\
\hline & 0.1 & Yes & Yes & Yes & Yes \\
\hline & 0.05 & Yes & Yes & Yes & Yes \\
\hline \multirow[t]{18}{*}{$s=4$} & 4 & No & No & No & No \\
\hline & 3.75 & No & No & No & No \\
\hline & 3.5 & No & No & No & No \\
\hline & 3.25 & No & No & No & No \\
\hline & 3 & No & No & No & No \\
\hline & 2.75 & No & No & No & No \\
\hline & 2.5 & No & No & No & No \\
\hline & 2.25 & No & No & No & No \\
\hline & 2 & Yes & No & No & No \\
\hline & 1.75 & Yes & No & No & No \\
\hline & 1.5 & Yes & No & Yes & Yes \\
\hline & 1.25 & Yes & Yes & Yes & Yes \\
\hline & 1 & Yes & Yes & Yes & Yes \\
\hline & 0.75 & Yes & Yes & Yes & Yes \\
\hline & 0.5 & Yes & Yes & Yes & Yes \\
\hline & 0.25 & Yes & Yes & Yes & Yes \\
\hline & 0.1 & Yes & Yes & Yes & Yes \\
\hline & 0.05 & Yes & Yes & Yes & Yes \\
\hline
\end{tabular}


TABLE 4

ADMISSIBILITY OF THE LOCAL MOMENT MATCHING METHOD WITH TWO OR THREE MOMENTS KNOWN, $\theta_{E X P}=0.6, \alpha_{G A M}=2, \tau_{G A M}=1, \alpha_{P A R}=4, \theta_{P A R}=5, \mu_{L N}=0, \sigma_{L N}=1.5$ and $N=10$.

\begin{tabular}{|c|c|c|c|c|c|}
\hline Upper limit & Span & Exponential & Gamma & Pareto & Log-Normal \\
\hline \multirow[t]{22}{*}{$s=3$} & 5 & No & No & No & No \\
\hline & 4.75 & No & No & No & No \\
\hline & 4.5 & No & No & No & No \\
\hline & 4.25 & No & No & No & No \\
\hline & 4 & No & No & No & No \\
\hline & 3.75 & No & No & No & No \\
\hline & 3.5 & No & No & No & No \\
\hline & 3.25 & No & No & No & No \\
\hline & 3 & No & No & No & Yes \\
\hline & 2.75 & No & No & No & Yes \\
\hline & 2.5 & No & No & No & Yes \\
\hline & 2.25 & No & No & Yes & Yes \\
\hline & 2 & Yes & No & Yes & Yes \\
\hline & 1.75 & Yes & No & Yes & Yes \\
\hline & 1.5 & Yes & No & Yes & Yes \\
\hline & 1.25 & Yes & Yes & Yes & Yes \\
\hline & 1 & Yes & Yes & Yes & Yes \\
\hline & 0.75 & Yes & Yes & Yes & Yes \\
\hline & 0.5 & Yes & Yes & Yes & Yes \\
\hline & 0.25 & Yes & Yes & Yes & Yes \\
\hline & 0.1 & Yes & Yes & Yes & Yes \\
\hline & 0.05 & Yes & Yes & Yes & Yes \\
\hline \multirow[t]{22}{*}{$s=4$} & 5 & No & No & No & No \\
\hline & 4.75 & No & No & No & No \\
\hline & 4.5 & No & No & No & No \\
\hline & 4.25 & No & No & No & No \\
\hline & 4 & No & No & No & No \\
\hline & 3.75 & No & No & No & No \\
\hline & 3.5 & No & No & No & No \\
\hline & 3.25 & No & No & No & No \\
\hline & 3 & No & No & No & No \\
\hline & 2.75 & No & No & No & No \\
\hline & 2.5 & No & No & No & No \\
\hline & 2.25 & No & No & No & No \\
\hline & 2 & Yes & No & No & No \\
\hline & 1.75 & Yes & No & No & No \\
\hline & 1.5 & Yes & No & Yes & Yes \\
\hline & 1.25 & Yes & Yes & Yes & Yes \\
\hline & 1 & Yes & Yes & Yes & Yes \\
\hline & 0.75 & Yes & Yes & Yes & Yes \\
\hline & 0.5 & Yes & Yes & Yes & Yes \\
\hline & 0.25 & Yes & Yes & Yes & Yes \\
\hline & 0.1 & Yes & Yes & Yes & Yes \\
\hline & 0.05 & Yes & Yes & Yes & Yes \\
\hline
\end{tabular}


TABLE 5

ADMISSIBILITY OF THE LOCAL MOMENT MATCHING METHOD ON EXPONENTIALLY DISTRIBUTED RISKS WITH PARAMETER $\theta$, TWO OR THREE MOMENTS KNOWN AND $N=5$.

\begin{tabular}{|c|c|c|c|c|c|}
\hline & Span & $\theta=0.2$ & $\theta=0.5$ & $\theta=1$ & $\theta=2$ \\
\hline \multirow[t]{14}{*}{$s=3$} & 3 & Yes & No & No & No \\
\hline & 2.75 & Yes & No & No & No \\
\hline & 2.5 & Yes & Yes & No & No \\
\hline & 2.25 & Yes & Yes & No & No \\
\hline & 2 & Yes & Yes & No & No \\
\hline & 1.75 & Yes & Yes & No & No \\
\hline & 1.5 & Yes & Yes & No & No \\
\hline & 1.25 & Yes & Yes & Yes & No \\
\hline & 1 & Yes & Yes & Yes & No \\
\hline & 0.75 & Yes & Yes & Yes & No \\
\hline & 0.5 & Yes & Yes & Yes & Yes \\
\hline & 0.25 & Yes & Yes & Yes & Yes \\
\hline & 0.1 & Yes & Yes & Yes & Yes \\
\hline & 0.05 & Yes & Yes & Yes & Yes \\
\hline \multirow[t]{14}{*}{$s=4$} & 3 & Yes & No & No & No \\
\hline & 2.75 & Yes & No & No & No \\
\hline & 2.5 & Yes & No & No & No \\
\hline & 2.25 & Yes & Yes & No & No \\
\hline & 2 & Yes & Yes & No & No \\
\hline & 1.75 & Yes & Yes & No & No \\
\hline & 1.5 & Yes & Yes & No & No \\
\hline & 1.25 & Yes & Yes & No & No \\
\hline & 1 & Yes & Yes & Yes & No \\
\hline & 0.75 & Yes & Yes & Yes & No \\
\hline & 0.5 & Yes & Yes & Yes & Yes \\
\hline & 0.25 & Yes & Yes & Yes & Yes \\
\hline & 0.1 & Yes & Yes & Yes & Yes \\
\hline & 0.05 & Yes & Yes & Yes & Yes \\
\hline
\end{tabular}

Table 10 displays the results obtained in this case. Contrarily to the continuous case, there seems now to exist "extremal" spans $\Delta_{\min }>0$ and $\Delta_{\max }>0$ for which, for all given span $\Delta>0$ (such that there is more than one interval $I_{k}$ in Section 3) and $\Delta_{\min } \leq \Delta \leq \Delta_{\max }$, the local moment matching method leads to a discretisation of the distribution of interest with positive masses. When discretisation is possible, we also give the first three moments of the approached discrete distributions (see Table 10). When using $s-1$ moments, the more $\Delta$ tends to $\Delta_{\min }$, the more the $s$ th moment tends to the true one. This was 
TABLE 6

ADMISSIBILITY OF THE LOCAL MOMENT MATCHING METHOD ON GAMMA DISTRIBUTED RISKS WITH PARAMETERS $(\alpha, \tau)$, TWO OR THREE MOMENTS KNOWN AND $N=5$.

\begin{tabular}{|c|c|c|c|c|c|c|c|}
\hline & \multirow{2}{*}{ Span } & \multicolumn{3}{|c|}{$\alpha=0.5$} & \multicolumn{3}{|c|}{$\alpha=1$} \\
\hline & & $\tau=0.25$ & $\tau=0.5$ & $\tau=1$ & $\tau=0.25$ & $\tau=0.5$ & $\tau=1$ \\
\hline \multirow[t]{14}{*}{$s=3$} & 3 & No & No & No & Yes & No & No \\
\hline & 2.75 & Yes & No & No & Yes & No & No \\
\hline & 2.5 & Yes & No & No & Yes & Yes & No \\
\hline & 2.25 & Yes & No & No & Yes & Yes & No \\
\hline & 2 & Yes & No & No & Yes & Yes & No \\
\hline & 1.75 & Yes & Yes & No & Yes & Yes & No \\
\hline & 1.5 & Yes & Yes & No & Yes & Yes & No \\
\hline & 1.25 & Yes & Yes & No & Yes & Yes & Yes \\
\hline & 1 & Yes & Yes & No & Yes & Yes & Yes \\
\hline & 0.75 & Yes & Yes & Yes & Yes & Yes & Yes \\
\hline & 0.5 & Yes & Yes & Yes & Yes & Yes & Yes \\
\hline & 0.25 & Yes & Yes & Yes & Yes & Yes & Yes \\
\hline & 0.1 & Yes & Yes & Yes & Yes & Yes & Yes \\
\hline & 0.05 & Yes & Yes & Yes & Yes & Yes & Yes \\
\hline \multirow[t]{14}{*}{$s=4$} & 3 & No & No & No & Yes & No & No \\
\hline & 2.75 & No & No & No & Yes & No & No \\
\hline & 2.5 & Yes & No & No & Yes & No & No \\
\hline & 2.25 & Yes & No & No & Yes & Yes & No \\
\hline & 2 & Yes & No & No & Yes & Yes & No \\
\hline & 1.75 & Yes & No & No & Yes & Yes & No \\
\hline & 1.5 & Yes & No & No & Yes & Yes & No \\
\hline & 1.25 & Yes & Yes & No & Yes & Yes & No \\
\hline & 1 & Yes & Yes & No & Yes & Yes & Yes \\
\hline & 0.75 & Yes & Yes & No & Yes & Yes & Yes \\
\hline & 0.5 & Yes & Yes & Yes & Yes & Yes & Yes \\
\hline & 0.25 & Yes & Yes & Yes & Yes & Yes & Yes \\
\hline & 0.1 & Yes & Yes & Yes & Yes & Yes & Yes \\
\hline & 0.05 & Yes & Yes & Yes & Yes & Yes & Yes \\
\hline
\end{tabular}

expected because, as the span decreases, the approached distribution tends to the true one.

\section{DisCUSSION}

In this paper, it appears that arithmetizing a risk by the local moment matching method with $s-1$ moments is equivalent to locally consider the discrete 
TABLE 7

ADMISSIBILITY OF THE LOCAL MOMENT MATCHING METHOD ON GAMMA DISTRIBUTED RISKS WITH PARAMETERS $(\alpha, \tau)$, TWO OR THREE MOMENTS KNOWN AND $N=5$.

\begin{tabular}{|c|c|c|c|c|c|c|c|}
\hline & \multirow{2}{*}{ Span } & \multicolumn{3}{|c|}{$\alpha=1.5$} & \multicolumn{3}{|c|}{$\alpha=2$} \\
\hline & & $\tau=0.25$ & $\tau=0.5$ & $\tau=1$ & $\tau=0.25$ & $\tau=0.5$ & $\tau=1$ \\
\hline \multirow[t]{14}{*}{$s=3$} & 3 & Yes & Yes & No & Yes & Yes & No \\
\hline & 2.75 & Yes & Yes & No & Yes & Yes & No \\
\hline & 2.5 & Yes & Yes & No & Yes & Yes & Yes \\
\hline & 2.25 & Yes & Yes & No & Yes & Yes & No \\
\hline & 2 & Yes & Yes & No & Yes & Yes & No \\
\hline & 1.75 & Yes & Yes & No & Yes & Yes & No \\
\hline & 1.5 & Yes & Yes & Yes & Yes & Yes & Yes \\
\hline & 1.25 & Yes & Yes & Yes & Yes & Yes & Yes \\
\hline & 1 & Yes & Yes & Yes & Yes & Yes & Yes \\
\hline & 0.75 & Yes & Yes & Yes & Yes & Yes & Yes \\
\hline & 0.5 & Yes & Yes & Yes & Yes & Yes & Yes \\
\hline & 0.25 & Yes & Yes & Yes & Yes & Yes & Yes \\
\hline & 0.1 & Yes & Yes & Yes & Yes & Yes & Yes \\
\hline & 0.05 & Yes & Yes & Yes & Yes & Yes & Yes \\
\hline \multirow[t]{14}{*}{$s=4$} & 3 & Yes & Yes & No & Yes & Yes & No \\
\hline & 2.75 & Yes & Yes & No & Yes & Yes & No \\
\hline & 2.5 & Yes & Yes & No & Yes & Yes & No \\
\hline & 2.25 & Yes & Yes & No & Yes & Yes & No \\
\hline & 2 & Yes & Yes & No & Yes & Yes & No \\
\hline & 1.75 & Yes & Yes & No & Yes & Yes & No \\
\hline & 1.5 & Yes & Yes & No & Yes & Yes & No \\
\hline & 1.25 & Yes & Yes & Yes & Yes & Yes & Yes \\
\hline & 1 & Yes & Yes & Yes & Yes & Yes & Yes \\
\hline & 0.75 & Yes & Yes & Yes & Yes & Yes & Yes \\
\hline & 0.5 & Yes & Yes & Yes & Yes & Yes & Yes \\
\hline & 0.25 & Yes & Yes & Yes & Yes & Yes & Yes \\
\hline & 0.1 & Yes & Yes & Yes & Yes & Yes & Yes \\
\hline & 0.05 & Yes & Yes & Yes & Yes & Yes & Yes \\
\hline
\end{tabular}

$s$-convex extremal risks with appropriate moments. This allows us to give simple and explicit criteria to find quickly when the local moment matching method will fail to produce a bona fide discrete probability distribution. Explicit conditions to be fulfilled have been derived here for the 1-, 2-, and 3-moment case.

To end with, let us mention that, in case a certain local moment sequence is not admissible, VILAR (2000) proposed a complementary approach based 
TABLE 8

AdmissibiLity OF THE LOCAL MOMENT MATCHING METHOD ON PARETO DISTRIBUTED RiskS WITH PARAMETERS $(\alpha, \theta)$, TWO OR THREE MOMENTS KNOWN AND $N=5$.

\begin{tabular}{|c|c|c|c|c|c|c|c|c|c|c|}
\hline & \multirow{2}{*}{ Span } & \multicolumn{3}{|c|}{$\theta=2$} & \multicolumn{3}{|c|}{$\theta=4$} & \multicolumn{3}{|c|}{$\theta=6$} \\
\hline & & $\alpha=4$ & $\alpha=5$ & $\alpha=6$ & $\alpha=4$ & $\alpha=5$ & $\alpha=6$ & $\alpha=4$ & $\alpha=5$ & $\alpha=6$ \\
\hline \multirow[t]{14}{*}{$s=3$} & 3 & No & No & No & No & No & No & No & No & No \\
\hline & 2.75 & No & No & No & No & No & No & No & No & No \\
\hline & 2.5 & No & No & No & No & No & No & No & No & No \\
\hline & 2.25 & No & No & No & No & No & No & Yes & No & No \\
\hline & 2 & No & No & No & No & No & No & Yes & Yes & No \\
\hline & 1.75 & No & No & No & Yes & No & No & Yes & Yes & Yes \\
\hline & 1.5 & No & No & No & Yes & No & No & Yes & Yes & Yes \\
\hline & 1.25 & No & No & No & Yes & Yes & No & Yes & Yes & Yes \\
\hline & 1 & No & No & No & Yes & Yes & Yes & Yes & Yes & Yes \\
\hline & 0.75 & Yes & No & No & Yes & Yes & Yes & Yes & Yes & Yes \\
\hline & 0.5 & Yes & Yes & Yes & Yes & Yes & Yes & Yes & Yes & Yes \\
\hline & 0.25 & Yes & Yes & Yes & Yes & Yes & Yes & Yes & Yes & Yes \\
\hline & 0.1 & Yes & Yes & Yes & Yes & Yes & Yes & Yes & Yes & Yes \\
\hline & 0.05 & Yes & Yes & Yes & Yes & Yes & Yes & Yes & Yes & Yes \\
\hline \multirow[t]{14}{*}{$s=4$} & 3 & No & No & No & No & No & No & No & No & No \\
\hline & 2.75 & No & No & No & No & No & No & No & No & No \\
\hline & 2.5 & No & No & No & No & No & No & No & No & No \\
\hline & 2.25 & No & No & No & No & No & No & No & No & No \\
\hline & 2 & No & No & No & No & No & No & Yes & No & No \\
\hline & 1.75 & No & No & No & No & No & No & Yes & No & No \\
\hline & 1.5 & No & No & No & No & No & No & Yes & Yes & No \\
\hline & 1.25 & No & No & No & Yes & No & No & Yes & Yes & Yes \\
\hline & 1 & No & No & No & Yes & Yes & No & Yes & Yes & Yes \\
\hline & 0.75 & No & No & No & Yes & Yes & Yes & Yes & Yes & Yes \\
\hline & 0.5 & Yes & Yes & No & Yes & Yes & Yes & Yes & Yes & Yes \\
\hline & 0.25 & Yes & Yes & Yes & Yes & Yes & Yes & Yes & Yes & Yes \\
\hline & 0.1 & Yes & Yes & Yes & Yes & Yes & Yes & Yes & Yes & Yes \\
\hline & 0.05 & Yes & Yes & Yes & Yes & Yes & Yes & Yes & Yes & Yes \\
\hline
\end{tabular}

on the nearness of local moment built in terms of linear programming. More precisely, if a certain sequence of $n$ local moments is not admissible, then the author proposes to determine the arithmetic distribution which conserves the $n-1$ first moments and has the nearest $n$th moment to the true one. Obviously, if the sequence of $n$ moments is admissible, then the solution coincides with the one obtained from the local moment matching method with $n$ moments. 
TABLE 9

ADMISSIBILITY OF THE LOCAL MOMENT MATCHING METHOD ON LOG-NORMALLY DISTRIBUTED RISKS WITH PARAMETERS $\mu=0$ AND $\sigma$, TWO OR THREE MOMENTS KNOWN AND $N=5$.

\begin{tabular}{|c|c|c|c|c|}
\hline & Span & $\sigma=0.5$ & $\sigma=1$ & $\sigma=1.5$ \\
\hline \multirow[t]{14}{*}{$s=3$} & 3 & No & No & No \\
\hline & 2.75 & No & No & No \\
\hline & 2.5 & No & No & No \\
\hline & 2.25 & No & No & Yes \\
\hline & 2 & No & Yes & Yes \\
\hline & 1.75 & No & Yes & Yes \\
\hline & 1.5 & No & Yes & Yes \\
\hline & 1.25 & No & Yes & Yes \\
\hline & 1 & No & Yes & Yes \\
\hline & 0.75 & Yes & Yes & Yes \\
\hline & 0.5 & No & Yes & Yes \\
\hline & 0.25 & No & Yes & Yes \\
\hline & 0.1 & No & No & Yes \\
\hline & 0.05 & No & No & Yes \\
\hline \multirow[t]{14}{*}{$s=4$} & 3 & No & No & No \\
\hline & 2.75 & No & No & No \\
\hline & 2.5 & No & No & No \\
\hline & 2.25 & No & No & No \\
\hline & 2 & No & No & No \\
\hline & 1.75 & No & No & No \\
\hline & 1.5 & No & No & Yes \\
\hline & 1.25 & No & Yes & Yes \\
\hline & 1 & No & Yes & Yes \\
\hline & 0.75 & No & Yes & Yes \\
\hline & 0.5 & No & Yes & Yes \\
\hline & 0.25 & No & Yes & Yes \\
\hline & 0.1 & No & Yes & Yes \\
\hline & 0.05 & No & Yes & Yes \\
\hline
\end{tabular}

\section{ACKNOWLEDGEMENTS}

The financial support of the Communauté française de Belgique under contract "Projet d'Actions de Recherche Concertées" ARC 04/09-320 is gratefully acknowledged.

\section{REFERENCES}

Courtois, C., Denuit, M. and Van Bellegem, S. (2006) Discrete $s$-convex extremal distributions:

Theory and applications. Applied Mathematics Letters, 19, 1367-1377. 
TABLE 10

LOCAL MOMENTS AND ADMISSIBILITY WITH THE HYPOTHETICAL CLAIM SEVERITY DISTRIBUTION GIVEN IN WALHIN \& PARIS (1998)

\begin{tabular}{|c|c|c|c|c|c|}
\hline & & Admissibility & $\mu_{1}$ & $\mu_{2}$ & $\mu_{3}$ \\
\hline True moments & & & 31.5 & 1401.8 & 71879.1 \\
\hline $\begin{array}{l}\text { Local moments } \\
\qquad s=2\end{array}$ & $\begin{array}{l}\Delta=1 \\
\Delta=5 \\
\Delta=10 \\
\Delta=15 \\
\Delta=20 \\
\Delta=25\end{array}$ & $\begin{array}{l}\text { Yes } \\
\text { Yes } \\
\text { Yes } \\
\text { Yes } \\
\text { Yes } \\
\text { Yes }\end{array}$ & $\begin{array}{l}31.5 \\
31.5 \\
31.5 \\
31.5 \\
31.5 \\
31.5\end{array}$ & $\begin{array}{l}1401.8 \\
1407 \\
1419 \\
1443 \\
1474 \\
1505\end{array}$ & $\begin{array}{l}71879.1 \\
72390 \\
73650 \\
76072.5 \\
79200 \\
81562.5\end{array}$ \\
\hline $\begin{array}{l}\text { Local moments } \\
\qquad s=3\end{array}$ & $\begin{array}{l}\Delta=1 \\
\Delta=5 \\
\Delta=10 \\
\Delta=15 \\
\Delta=16 \\
\Delta=16.7499 \\
\Delta=16.7500 \\
\Delta=17 \\
\Delta=18 \\
\Delta=19 \\
\Delta=19.7532 \\
\Delta=19.7533 \\
\Delta=20 \\
\Delta=25\end{array}$ & $\begin{array}{l}\text { No } \\
\text { No } \\
\text { No } \\
\text { No } \\
\text { No } \\
\text { No } \\
\text { Yes } \\
\text { Yes } \\
\text { Yes } \\
\text { Yes } \\
\text { Yes } \\
\text { No } \\
\text { No } \\
\text { No }\end{array}$ & $\begin{array}{l}31.5 \\
31.5 \\
31.5 \\
31.5 \\
31.5\end{array}$ & $\begin{array}{l}1401.8 \\
1401.8 \\
1401.8 \\
1401.8 \\
1401.8\end{array}$ & $\begin{array}{l}71650.22 \\
71639.7 \\
71523 \\
71335.5 \\
71183.75\end{array}$ \\
\hline $\begin{array}{l}\text { Local moments } \\
\qquad s=4\end{array}$ & $\begin{array}{l}\Delta=1 \\
\Delta=5 \\
\Delta=7.4514 \\
\Delta=8.6402 \\
\Delta=10 \\
\Delta=15 \\
\Delta=16 \\
\Delta=17 \\
\Delta=18 \\
\Delta=19 \\
\Delta=20\end{array}$ & $\begin{array}{l}\text { No } \\
\text { No } \\
\text { Yes } \\
\text { Yes } \\
\text { No } \\
\text { No } \\
\text { No } \\
\text { No } \\
\text { No } \\
\text { No } \\
\text { No }\end{array}$ & $\begin{array}{l}31.5 \\
31.5\end{array}$ & $\begin{array}{l}1401.8 \\
1401.8\end{array}$ & $\begin{array}{l}71879.1 \\
71879.1\end{array}$ \\
\hline
\end{tabular}

Denuit, M. and LefÈvre, Cl. (1997) Some new classes of stochastic order relations among arithmetic random variables, with applications in actuarial sciences. Insurance: Mathematics and Economics, 20, 197-214.

Denuit, M., De Vylder, F.E. and Lefèvre, Cl. (1999) Extremal generators and extremal distributions for the continuous s-convex stochastic orderings. Insurance: Mathematics and Economics, 24, 201-217. 
Denuit, M., Lefève, Cl. and Mesfioui, M. (1999) On $s$-convex stochastic extrema for arithmetic risks. Insurance: Mathematics and Economics, 25, 143-155.

Gerber, H.U. (1982) On the numerical evaluation on the distribution of aggregate claims and its stop-loss premiums. Insurance: Mathematics and Economics, 1, 13-18.

HÜRLIMANN, W. (2005) Improved analytical bounds for gambler's ruin probability. Methodology and Computing in Applied Probability, 7, 79-95.

Karlin, S. and Studden, W.J. (1966) Tchebycheff Systems with Applications in Analysis and Statistics. Wiley Interscience, New York.

Panjer, H.H. and Lutek, B.W. (1982) Practical aspects of stop-loss calculations. Insurance: Mathematics and Economics, 2, 159-177.

VILAR, J.L. (2000) Arithmetization of distributions and linear goal programming. Insurance: Mathematics and Economics, 27, 113-122.

Walhin, J.F. and PARIS, J. (1998) On the use of equispaced discrete distributions. ASTIN Bulletin, 28, 241-255.

\section{CindY COURTOIS}

Institut des Sciences Actuarielles

Université Catholique de Louvain

rue des Wallons 6

B-1348 Louvain-la-Neuve

Belgium

\section{Michel DenUit}

Institut de Statistique et Institut des Sciences Actuarielles

Université Catholique de Louvain

rue des Wallons 6

B-1348 Louvain-la-Neuve

Belgium 